\title{
Cardiac Autonomic Control in Adolescents with Primary HYPERTENSION
}

\author{
Z. Havlíceková1, ${ }^{1}$, I. Tonhajzerováa ${ }^{3}$ A. Jurko Jr. ${ }^{4}$, M. Jesenák ${ }^{1,2}$, P. Durdík ${ }^{1,2}$, S. Nosál1,2, K. Zelenák ${ }^{5}$, \\ M. Antosová 6 , P. Bánovcin 1,2 \\ Department of Pediatrics, ${ }^{2}$ Center of Experimental and Clinical Respirology, and \\ ${ }^{3}$ Institute of Physiology, Jessenius School of Medicine, Comenius University, Martin, Slovakia; \\ ${ }^{4}$ Pediatric Cardiological Private Office, Martin, Slovakia; ${ }^{5}$ Department of Radiology, University Hospital, Martin, Slovakia; \\ 'Institute of Pharmacology, Comenius University, Jessenius School of Medicine, Martin, Slovakia
}

\begin{abstract}
Background: Impairment in cardiovascular autonomic regulation participates in the onset and maintenance of primary hypertension.

Objective: The aim of the present study was to evaluate cardiac autonomic control using long-term heart rate variability (HRV) analysis in adolescents with primary hypertension.

Subjects and methods: Twenty two adolescent patients with primary hypertension (5 girls/17 boys) aged 1419 years and 22 healthy subjects matched for age and gender were enrolled. Two periods from 24-hour ECG recording were evaluated by HRV analysis: awake state and sleep. HRV analysis included spectral power in low frequency band (LF), in high frequency band (HF), and LF/HF ratio.
\end{abstract}

Results: In awake state, adolescents with primary hypertension had lower HF and higher LF and LF/HF ratio. During sleep, HF was lower and $\mathrm{LF} / \mathrm{HF}$ ratio was higher in patients with primary hypertension.

Conclusions: A combination of sympathetic predominance and reduced vagal activity might represent a potential link between psychosocial factors and primary hypertension, associated with increased cardiovascular morbidity.

Key words: primary hypertension, cardiac autonomic control, heart rate variability, adolescents

\section{INTRODUCTION}

Hypertension is one of the most serious human health problems. The prevalence of hypertension in childhood is increasing. The primary hypertension is closely linked to psychosocial characteristics; therefore, it is included into psychosomatic diseases [1]. In addition, adolescent primary hypertension is associated with higher risk for coronary artery disease and cardiovascular mortality in adult life. The autonomic nervous system is known to play a major role in the interaction of the cardiovascular and central nervous systems, and in other regulatory systems. It is expected that progressive impairment in autonomic regulation participates in the initiation and maintenance of primary hypertension, in particular in children and adolescents. Cardiac function is a sensitive to autonomic inputs; thus, a study of the cardiac autonomic control can be used for the assessment of primary hypertension.
The heart rate variability (HRV), i.e., the oscillations of heart rate around the mean value, is caused by variations in the input to the sinus node from the autonomic nervous system. Multiple mechanisms underlie HRV - parasympathetic activity at high frequency (HF) band reflects mainly respiratory sinus arrhythmia (RSA) and sympathetic activity at low frequency (LF) band reflects mainly baroreceptor activity. A long-term HRV analysis can provide information about the dependence of cardiac autonomic activity on day/night rhythm in primary hypertension. Guzzetti et al. [2], using the 24-hour HRV analysis, concluded that primary hypertension is characterized by higher sympathetic activity (greater LF). On the other side, other studies emphasize a contribution of parasympathetic activity in primary hypertension development [3]. Thus, the information on long-term heart rate variability is limited and somehow controversial.

In the present study, we set out to examine the cardiac autonomic control during awake state and sleep using a long-term HRV analysis in adolescents with primary hypertension.

\section{Subjects AND Methods}

The study was approved by the Ethics Committee of Jessenius Medical Faculty, Comenius University in Martin, Slovakia. Twenty-two subjects - 5 girls and 17 boys (aged 14-19 years, mean age $16 \pm 2$ years) with untreated primary hypertension were enrolled in the study. Primary hypertension was defined according to data recommended for hypertension diagnosis in childhood using 24-hour ambulatory blood pressure monitoring (ABPM) (Click Holter Recorder, Cardioline, Italy) with the mean systolic and/or diastolic blood pressure $\geq 95$ th percentile adjusted for height [4]. The diagnosis of primary hypertension was based on the examinations resulting in exclusion of secondary etiology of hypertension (e.g., renal, vascular, endocrine diseases, etc.).

All subjects with primary hypertension underwent 24-hour ABPM with nocturnal dip (more than $10 \%$ of the daytime blood pressure) in all hypertonics. A control group consisted of adolescents matched for age and gender (5 girls and 17 boys, mean age $17 \pm 1$ years). All probands were non-smokers, not taking drugs and substances influencing cardiovascular sys- 
tem (i.e., caffeine, alcohol), and they had no evidence of mental or other diseases.

A 24-h continuous ECG monitoring started in the morning (8 a.m.). All subjects carried out normal daily activities - school lessons, afternoon rest, preparation for next day school lessons, moderate physical activity, and sleep at night (after 10 p.m.). The ECG monitoring was finished next morning at 8 a.m.

\section{Heart Rate VARiability (HRV) ANALysis}

Artefacts in the 24-h ECG recording were eliminated using a recognition algorithm and also manually. The Hanning window was used for minimalization of spectral leakage. The HRV spectral analysis was performed using Fast Fourier transformation algorithm. Two periods from the 24-h ECG recording were selected for HRV analysis: awake state lasting from 9 a.m. to 1 p.m. reflecting daily activities and sleep lasting from midnight to $4 \mathrm{a} . \mathrm{m}$. The following parameters were evaluated:

LF - spectral power in low frequency band (0.04 $0.15 \mathrm{~Hz}$ )

HF -spectral power in high frequency band $(0.15-$ $0.4 \mathrm{~Hz})$

$\mathrm{LF} / \mathrm{HF}$ - ratio of low-high frequency powers

Spectral powers were expressed in normalized units (NU), which represent the relative value of each power component in proportion to the total power minus the very low frequency (VLF) component $(\mathrm{LF}=\mathrm{LF}$ $* 100$ / (total power - VLF). The representation of LF and HF in normalized units emphasizes the controlled and balanced behavior of the two branches of the autonomic nervous system. Moreover, the normalization tends to minimize the effects of the changes in total power on the values of LF and HF components. The HF power is determined mainly by parasympathetic activity (respiratory sinus arrhythmia) and the LF power is a reflection of both sympathetic and parasympathetic activities. Some studies suggest that LF, when expressed in normalized units, is a quantitative marker of sympathetic modulations. Moreover, the LF/HF ratio is considered an index of sympathovagal balance [5].

\section{STATISTICAL ANALYSIS}

The data are expressed as means \pm SE. The Lilliefors test was used for the determination of gaussian/nongaussian distribution. The Mann-Whitney U test and Wilcoxon test were used for between-groups comparison (hypertension vs. control; awake state vs. sleep). $\mathrm{P} \leq 0.05$ was considered as significant.

\section{RESULTS}

\section{Primary Hypertension vs. CONTROL}

The HF was significantly lower in adolescents with primary hypertension in both awake and sleep states, compared with those in controls $(\mathrm{P}=0.018$ and $\mathrm{P}=0.004$, respectively). The $\mathrm{LF}$ was higher in awake state $(\mathrm{P}=0.031)$, but did not change appreciably in sleep, in primary hypertension compared with con- trols. The LF/HF ratios were significantly higher in adolescents with primary hypertension in both awake and sleep states, compared with those controls $(\mathrm{P}=0.031$ and $\mathrm{P}=0.002$, respectively) (Table 1$)$.

\section{Awake State vs. Sleep}

The HF was significantly higher and LF/HF ratio was significantly lower during sleep in both groups $(\mathrm{P}=0.001)$. There were no significant differences in LF between the awake and sleep states in either primary hypertension or control group (Table 1).

Table 1. Frequency domain of HRV in subjects with primary hypertension and in the control group.

\begin{tabular}{lccc}
\hline & $\begin{array}{c}\text { Primary } \\
\text { Hypertension }\end{array}$ & Control & P \\
\hline \multicolumn{4}{c}{ Awake state } \\
LF & $38.12 \pm 1.05$ & $33.79 \pm 1.42$ & 0.031 \\
HF & $34.63 \pm 1.26$ & $37.71 \pm 1.12$ & 0.018 \\
LF/HF & $1.28 \pm 0.05$ & $0.91 \pm 0.04$ & 0.002 \\
& \multicolumn{3}{c}{ Sleep } \\
LF & $35.52 \pm 1.17$ & $32.69 \pm 0.90$ & NS \\
HF & $48.17 \pm 1.98$ & $54.57 \pm 0.75$ & 0.004 \\
LF/HF & $0.78 \pm 0.06$ & $0.60 \pm 0.02$ & 0.014 \\
\hline
\end{tabular}

Values are means \pm SE; LF - spectral power in low frequency band and HF - spectral power in high frequency band in normalized units $(\mathrm{NU})$.

\section{Discussion}

The basic finding of the present study was a decrease in parasympathetic HF activity during awake state and sleep, and an increase in sympathetic LF activity during awake state in adolescents with primary hypertension. These results indicate defective vagal regulation of the sinoatrial node associated with the shift of sympathovagal balance toward a sympathetic dominance (higher LF/HF) during day and night.

Studies concerning cardiovascular dysregulation in primary hypertension emphasize the role of a sympathetic branch of the autonomic nervous system. Enhanced sympathetic activity is widely accepted as one of the fundamental mechanisms leading to primary hypertension and may already be determined in prehypertensive subjects [6]. In addition, a close relationship between psychosocial characterics and primary hypertension is presumed. Some authors assume that excessive cardiovascular reactivity to psychological stress may have a causal mechanistic role in primary hypertension [7]. Thus, stress may affect major regulatory systems, in particular the autonomic nervous system, leading to inappropriately elevated sympathetic drive contributing to higher blood pressure. Some children with primary hypertension are characterized by typical psychological features, e.g., perfectionism or anxiety leading to chronic stress, which, in consequence, affects cardiac autonomic regulation [7]. Kamada et al. [8] found a shift of sympathovagal balance 
(higher LF/HF) using of the HRV analysis in A type subjects (competitive, ambitious, intensive life-style, faster breathing) compared with B type subjects (relaxing behaviour, deep breathing) [8]. The mechanisms by which psychosocial factors increase the risk of cardiovascular diseases, including hypertension, are numerous and complex; however, sympathetic overactivity seems to play a pivotal role $[9,10]$. Our results are in accordance with these studies indicating the sympathetic overactivity in primary hypertonics.

Importantly, some authors emphasize the contribution of the parasympathetic modulation in primary hypertension $[9,10]$, but its role is less clear. Some studies revealed lower vagal activity in young people with primary hypertension $[6,11]$. Others revealed a significant decrease in parameters representing vagal tone during 5-min periods not only immediately preceding or following blood pressure elevations, but also 10 and $20 \mathrm{~min}$ before these episodes. Moreover, low frequency component of HRV was significantly lowered 10 min before and immediately after the recording of blood pressure elevation. These results suggest that among various pathogenic mechanisms of spontaneous blood pressure elevations, sudden vagal withdrawal should be taken into account [12]. Our results confirm a decrease of the parasympathetic activity in primary hypertension.

The cardiac autonomic dysregulation in primary hypertension could be a result of the abnormal central activity and of alterations in effectors (heart, vessels) [3], but the exact mechanisms are still unclear. Moreover, patients with early primary hypertension may have a hyperkinetic pattern of hemodynamics which is characterized by higher cardiac output and higher heart rate and blood pressure [13]. It seems that a combination of sympathetic predominance and lower vagal activity can be a result of the multifactorial pathomechanisms leading to chronic elevation of blood pressure in adolescents, including the above-mentioned psychosocial factors. Thus, stress management could exert advantageous influence on hypertension prevention. It seems worth emphasizing the importance of the non-pharmacological (e.g., relaxation or physical activity) and psychosocial (e.g., decreasing anxiety or hyperreactivity to stressors) treatments of primary hypertension which may lessen cardiac autonomic dysregulation $[10,14]$.

In conclusion, our study has revealed a combination of sympathetic predominance and vagal withdrawal indicating impaired cardiac autonomic regulation during day and night in adolescents with primary hypertension. Importantly, autonomic imbalance may be the final common pathway of numerous diseases and conditions associated with increased morbidity and mortality of hypertension [15].

Acknowledgments: Supported by Grant of Ministry of Health and European Regional Development Fund No 2006/38UK-05. We thank M. Kollarik of John Hopkins University School of Medicine in Baltimore for helpful comments on the manuscript.

Conflicts of interest: The authors reported no conflicts of interest in relation to this article.

\section{REFERENCES}

[1] Dubiel J, Dukalska M, Bilewicz-Wyrozumska T, Skierska A. Characteristics of psychological factors participating in the etiopathogenesis of primary arterial hypertension of children and adolescents. Wiad Lek 2005; 58: 589-94.

[2] Guzzetti S, Dassi S, Pecis M et al. Altered pattern of circadian neural control of heart period in mild hypertension. J Hypertens 1991; 9: 831-8.

[3] Javorka K. Heart rate variability and essential hypertension. In Heart rate variability, mechanisms, evaluation and clinical use. K Javorka (ed). Martin, Osveta, 2008, pp. 133-134.

[4] Soergel M, Kirschstein M, Buchs CH, Danne T, Gellermann J, Holl R, Krull F, Reichert H, Reusz GS, Rascher W. Oscillometric twenty-four-hour ambulatory blood pressure values in healthy children and adolescents: A multicenter trial including 1141 subjects. J Pediatrics 1997; 130: 178-184.

[5] Task Force of European Society of Cardiology and The North American Society of Pacing and Electrophysiology: Heart rate variability. Standards of measurement, physiological interpretation, and clinical use. Eur Heart J 1996; 17: 354-381.

[6] Lucini D, Mela GS, Malliani A, Pagani M. Impairment in cardiac autonomic regulation preceding arterial hypertension in humans. Circulation 2002; 106: 2673-2679.

[7] Matthews K, Salomon K, Bradz SS, Allen MT. Cardiovascular reactivity to stress predicts future blood pressure in adolescence. Psychosom Med 2003; 65: 410-415.

[8] Kamada T, Sato N, Miyake S, Kumashiro M, Monou H. Power spectral analysis of heart rate variability in Type As during solo and competitive mental arithmetic task. J Psychosom Res 1992; 36: 543-551.

[9] Lucini D, DiFede G, Parati G, Pagani M. Impact of chronic psychosocial stress on autonomic cardiovascular regulation in otherwise healthy subjects. Hypertension 2005; 46: 1201-1206.

[10] Lucini D, Riva S, Pizzinelli P, Pagani M. Stress management at the worksite: Reversal of symptoms profile and cardiovascular dysregulation. Hypertension 2007; 49: 291 297.

[11] Mussalo H, Vanninen E, Ikäheimo R, Laitinen T, Laakso M, Länsimies E, Hartikainen J. Heart rate variability and its determinants in patients with severe or mild essential hypertension. Clin Physiol 2001; 21: 594-604.

[12] Dabrowska B, Dabrowski A, Skrobowski A. Parasympathetic withdrawal precedes spontaneous blood pressure elevations in women with primary hypertension. Cardiology 1996; 87: 119-124.

[13] Schneider GM, Jacobs DW, Gevirtz RN, O'Connor DT. Cardiovascular haemodynamic response to repeated mental stress in normotensive subjects at genetic risk of hypertension: evidence of enhanced reactivity, blunted adaptation and delayed recovery. J Hum Hypertens 2003; 17: 829-840.

[14] Matthews KA, Salomon K, Brady SB, Allen MT. Cardiovascular Reactivity to Stress Predicts Future Blood Pressure. Adolescence. Psychosom Med 2003; 65: 410-415.

[15] Thayer JF, Lane RD. The role of vagal function in the risk for cardiovascular disease and mortality. Biol Psychol 2007; 74: 224-242.

Corresponding author:

Zuzana Havlíceková, M.D., Ph.D.

Department of Paediatrics, Jessenius School of Medicine

Kollarova 2 St.

03659 Martin, Slovakia

Phone: +421434203254

Fax: +421434222678

zhavlicekova@gmail.com 